\title{
Risk Factors and Complications Associated with Carotid Artery Stenting: A Retrospective Study
}

\author{
Yo Seob Shin, Tae-Sik Gong, Hyo-Joon Kim, Min Park \\ Department of Neurosurgery, Presbyterian Medical Center, Jeonju, Republic of Korea
}

Corresponding author:

Tae-Sik Gong

Department of Neurosurgery,

Presbyterian Medical Center, 365,

Seowon-ro, Wansan-gu, Jeonju

54987, Republic of Korea

Tel: +82-63-230-1420

Fax: +82-63-230-1429

E-mail: nskts@hanmail.net

Received: August 15, 2019

Revised: August 26, 2019

Accepted: August 30, 2019
Objective: The use of selective carotid stents to reduce ipsilateral stroke in patients with cerebral artery stenosis has been increasing recently. The insertion of carotid stents can cause complications of embolism, and many efforts are involved to reduce such effects. This study investigated the risk factors and complications by examining 125 cases that underwent carotid artery stenting (CAS) in a single center in the past 5 years. Methods: The prognosis of 125 patients who underwent CAS in the past 5 years was evaluated by considering the following 2 outcomes: postprocedure symptomatic events and stroke or other vascular problems (SOVP). The prognosis also considered the following: non-modifiable factors, such as age and sex; modifiable factors, such as a smoking history, hypertension, and diabetes; and magnetic resonance imaging changes that occurred after CAS. Results: In this study, an age above 80 years $(p=0.031)$ and a history of cerebrovascular accidents $(p=0.016)$ were identified as the significant risk factors for the incidence of stroke among patients after stent insertion. The presence of a distal filter device and the type of maintenance therapy administered before and after CAS (aspirin, clopidogrel, or aspirin+clopidogrel) exhibited no significant effects on post-procedure outcomes. Conclusion: This study indicated that $2.6 \%$ of stroke symptoms are directly related to CAS and that $3.2 \%$ of SOVP occurred within 30 days after the procedure. Patients above the age of 80 or with a history of stroke should be fully informed of the risk factors and side effects of CAS procedures.

Key Words: Carotid arteries; Risk factors; Stents; Stroke

\section{INTRODUCTION}

According to data by World Health Organization ${ }^{13)}$, stroke is the third major cause of death worldwide. Severe carotid stenosis accounts for $20 \%$ of known etiologies of stroke; therefore, early diagnosis of lesions and prompt treatment are important to reduce the serious side-effects of stroke ${ }^{8)}$. Carotid artery stenosis has been directly correlated with ipsilateral stroke".

Recently, several studies have concluded that carotid artery stenting (CAS) and conventional endarterectomy have similar effects for treating stroke, and a superior method is yet to be determined $^{2,8,13,14)}$. To prevent cerebral infarction in patients with severe carotid stenosis, balloon angioplasty and percutaneous angioplasty with stenting is increasingly performed because of its minimal invasiveness.

Complications after stent insertion can be divided into treatment-related acute complications and post-procedure chronic complications. Factors affecting these complications include non-modifiable risk factors, such as age and sex, and modifiable risk factors, such as smoking history, hypertension, and diabetes. The risk of developing an embolism during stent release is the major complication associated with $\mathrm{CAS}^{8)}$. The use of an embolic protection device (EPD) is recommended to reduce the incidence of acute distal complications due to micro-embolism ${ }^{3,7)}$.

Chronic complications associated with stent insertion include ipsilateral cerebral infarction; cardiovascular problems, such as angina, myocardial infarction, and iliac artery stenosis. Most of these chronic complications can be prevented by the administration of proper medications.

In this study, we investigated various risk factors affecting the post-procedure complications, outcomes, and methods for improvement of outcomes after CAS. We established and evaluated 2 endpoints to achieve our objective - Postprocedure symptomatic event and incidence of stroke or other vascular problem (SOVP).

\section{MATERIALS AND METHODS}

\section{Patients}

In this retrospective cohort study, we included patients who underwent CAS at a single center; Presbyterian medical center in Jeonju, South Korea, between a 5-year period from January 
2013 to December 2017. A single surgeon with over 10 years of experience performed all surgeries as a single operative procedure without any learning curve. The requirement for informed consent was waived because of the retrospective study design.

\section{CAS}

CAS was performed under local anesthesia. After induction of anesthesia, the right femoral artery was punctured with a 16-gauge needle. After insertion of the guide wire, an 8-Fr sheath was inserted using the Seldinger method. The carotid artery approach used Headhunter catheters, DAV, Simmons, or Newton catheters were used when not suitable. The 8-Fr guiding catheter was inserted into the lesion, proximal to the common carotid artery. The distal filter device FilterWire (Spider $\mathrm{FX}^{\mathrm{TM}}$; Covidien, Mansfield, MA, USA), an EPD, was inserted approximately 3 to $5 \mathrm{~cm}$ above the end point of the significant stenosis using the delivery sheath. Using a 4.0 to $6.0 \mathrm{~mm}$ balloon catheter, the stenotic lesion was pre-expanded. Self-extendable stents; 7.0 to $10.0 \mathrm{~mm}$ diameter and 30 to $40 \mathrm{~mm}$ length, depending on the shape and length of the lesion were selected and inserted to adequately cover the lesion. One types of stents were - protégé stent (ev3, Endovascular Inc., Plymouth, MN, USA). Cases in which the lesion did not extend sufficiently after stenting, the unexpanded part inside the stent was subsequently postexpanded using balloons. To manage the risk or presentation of bradycardia during balloon expansion, atropine or dopamine was injected based on the surgeon's judgment. After insertion of the stent, the FilterWire was removed using the provided retrieval sheath.

\section{Evaluation of Prognosis after CAS}

After stent insertion, the patients were admitted to intensive care unit and the symptoms were monitored until postoperative day 1 to control their blood pressure. On postoperative day 1 , diffusion-weighted magnetic resonance imaging (MRI) of the brain were obtained to assess brain lesions, and subsequently, the patients were shifted to the general ward. To evaluate patient prognosis, symptomatic events or SOVP, or postoperative changes on MRI were investigated immediately within $24 \mathrm{hr}$.

Subsequently, all patients who underwent CAS were retrospectively investigated based on their outpatient chart and examined for incidences of cardiovascular problems during the outpatient follow-up period until June 2019 or until loss of follow-up. Postoperative complications included cerebral infarction, transient ischemic attack, myocardial infarction, iliac artery stenosis, and death.

\section{Statistical Analyses}

The $\chi^{2}$ test and Fisher's exact probability test were used to confirm the relationship between stroke occurrence after stent insertion and the multiple risk/prognosis factors. Binary logistic regression was used for multivariate analysis among the factors. All statistical analyses were performed using SPSS version 20.0 (SPSS Inc., Chicago, IL, USA); p-values less than or equal to 0.05 were considered statistically significant.

\section{RESULTS}

During the 5-year duration of the study, CAS was performed 139 times in 128 patients with stenosis. Three patients among them were excluded because they did not undergo postprocedure brain MRI scan; 11 people simultaneously underwent left and right sided stent insertion within a week of CAS. Eventually, 125 patients were included in the study; 116 men (92.8\%) and 9 women (7.2\%), and all 11 patient who underwent bilateral stenting were men. The median age of the entire patient cohort was 71.4 (40-90) years. Ninety-nine (85.3\%) patients underwent CAS in the carotid bulb and $17(14.7 \%)$ in the distal carotid artery. Fifty-seven patients were diagnosed with cerebral infarction before angiography and stent insertion; 2 patients were diagnosed with transient ischemic attack; and 11 patients had cerebral hemorrhage. Among the 113 cases, a distal EPD was used in $90.4 \%$ of the patients.

Among patient history and underlying comorbidities, 104 patients had hypertension, 51 patients had diabetes, 66 patients had hyperlipidemia, 28 patients previously had cerebrovascular diseases, and 58 patients had a history of smoking. Single-drug and combination therapies of aspirin or clopidogrel were used as preprocedure medications and postprocedure maintenance drugs for all patients. CAS was successful in all 125 patients

Within $24 \mathrm{hr}$ of CAS, diffusion-weighted MRI was performed for all patients to assess acute period complications. Among them, 95 people (81.9\%) showed various changes on MRI because of embolism, and 3 of them showed neurological symptoms, such as dysphasia and hemiplegia.

Chronic complications, including SOVP, were observed in $23(19.8 \%)$ patients during the follow-up period.

Six patients had cerebral infarction, 9 patients underwent percutaneous coronary intervention, 3 of them were in patients who were treated for transient ischemic attack, 4 patients were diagnosed with iliac artery stenosis, and 1 patient died. The deceased was found dead-on-arrival 3 years after CAS, and the exact cause of death was unknown. Among those with chronic complications, 4 (3.2\%) patients showed major adverse events within 30 days of stent insertion. In previous clinical trials, such as ARCHeR 2, ARCHeR 3, BEACH, MAVErIC 1, MAVErIC 2, and CREATE, those with major postoperative complications averaged $6.3 \%$ of the cohort, twice the value of the present study ${ }^{11)}$. The rates of acute and chronic complications in this study are shown in Table 1.

We evaluated the statistical significance for each postoperative risk factor and changes on MRI, and for the postopera- 
tive SOVP observed during the follow-up observation period (Table 2). Stent insertion in older patients (above 80 years of age) significantly correlated with events of SOVP $(\mathrm{p}=0.031)$; this trend was similar to that reported for elderly patients

Table 1. Post-procedural acute and chronic clinical outcome

\begin{tabular}{lc}
\hline \hline & $\mathrm{n}(\%)$ \\
\hline Post stent event (MRI, <24 hour) & \\
MRI signal change & $95(81.9)$ \\
Symptomatic event & $3(2.6)$ \\
Long term event & $23(19.8)$ \\
$\mathrm{PCl}$ & $9(7.8)$ \\
Cerebral infarction & $6(5.2)$ \\
TIA & $3(2.6)$ \\
lliac A stenosis & $4(3.4)$ \\
Death & $1(0.9)$ \\
\hline
\end{tabular}

MRI: magnetic resonance imaging; $\mathrm{PCl}$ : percutaneous coronary intervention; TIA: transient ischemic attack. with stroke.

In addition, history of cerebrovascular accident, including hemorrhage and infarction, significantly affected SOVP ( $\mathrm{p}=0.016)$. Other clinical features, such as hypertension, diabetes, hyperlipidemia, the type and number of maintenance drugs used, and location of lesions did not significantly affect the postoperative outcomes after stent insertion. Moreover, the use of distal filter EPD to prevent incidences of postoperative embolism and postoperative changes on MRI did not differ significantly from the occurrence of complications after CAS.

\section{DISCUSSION}

In this single-center study, we compared the factors that affected the postoperative outcomes of CAS using a single procedure in more than 100 carotid regions with the same type of stent. Our study design ensured the maximal elimination of bias with regard to the surgeon, hospital, and type of instrument used. CAS is performed to prevent the recurrence of cerebral infarction, and the use of carotid stents is increasing and

Table 2. Statistical significance of each factor and the long-term prognosis after the stent

\begin{tabular}{|c|c|c|c|c|c|}
\hline \multirow{2}{*}{ Variables } & \multicolumn{3}{|c|}{ Univariate } & \multicolumn{2}{|c|}{ Multivariate } \\
\hline & $\mathrm{n}(\%)$ & No. of events & p-value & $\operatorname{Exp}(b)$ & p-value \\
\hline Sex (male) & $116(92.8)$ & 20 & 0.831 & & \\
\hline Age $(>80)$ & $26(20.8)$ & 6 & 0.031 & 0.366 & 0.043 \\
\hline Left & $63(50.4)$ & 12 & 0.511 & & \\
\hline Location (carotid bulb) & $96(76.8)$ & 18 & 0.836 & & \\
\hline \multicolumn{6}{|l|}{ Stenosis } \\
\hline$>70 \%$ & $117(93.6)$ & 19 & 0.523 & & \\
\hline$>90 \%$ & 24 (19.2) & 6 & 0.281 & & \\
\hline \multicolumn{6}{|l|}{ Pre-stent diagnosis } \\
\hline Cerebral infarction & $56(44.8)$ & 11 & 0.303 & & \\
\hline Any type of stroke & 69 (55.2) & 14 & 0.099 & & \\
\hline Protective filter & $113(90.4)$ & 22 & 0.224 & & \\
\hline \multicolumn{6}{|l|}{ Comorbidities } \\
\hline Hypertension & $104(83.2)$ & 17 & 0.811 & & \\
\hline Diabetes & 51 (40.8) & 7 & 0.355 & & \\
\hline Hyperlipidemia & $66(52.8)$ & 11 & 0.674 & & \\
\hline Previous CVA & $28(22.4)$ & 8 & 0.016 & 0.337 & 0.015 \\
\hline Heart failure & $29(23.2)$ & 4 & 0.898 & & \\
\hline Atrial fibrillation & $5(4.0)$ & 0 & 0.362 & & \\
\hline Smoking & $58(46.4)$ & 9 & 0.525 & & \\
\hline Post MRI change & $95(76.0)$ & 18 & 0.260 & & \\
\hline \multicolumn{6}{|l|}{ Maintenance drug } \\
\hline Aspirin & $123(98.4)$ & 22 & 0.545 & & \\
\hline Clopidogrel & $124(99.2)$ & 21 & 0.757 & & \\
\hline Aspirin+Clopidogrel & $110(88.0)$ & 19 & 0.922 & & \\
\hline
\end{tabular}

CVA: cerebrovascular accident; MRI: magnetic resonance imaging. 
has the potential to replace carotid endarterectomy; how- ever, many studies reported no difference in the long-term prognosis between CAS and endarterectomy ${ }^{2}$. With increasing application of CAS, the use of EPD has been gradually expanded to facilitate the reduction in the cases of distal embolism, a known complication of CAS. In this study, we evaluated various risk factors and distal filter EPD to assess the outcomes after CAS. Symptomatic stroke during CAS occurred at a rate of 2.6\%, lower than that reported previously ${ }^{14)}$.

Previous studies have shown that old age is an important prognostic factor in patients undergoing $\mathrm{CAS}^{5,6)}$. In this study, age above 80 years $(\mathrm{p}=0.031)$ and history of cerebrovascular disease $(p=0.016)$ had a statistically significant effect on the occurrence of cerebral infarction after stenting. The age groups of 60 and 70 showed no significant difference with regard to the factors affecting outcomes after CAS; only patients 80 years and older showed an increase in the incidence of SOVP. As a non-modifiable risk factor for stroke, this result is more meaningful than the stent itself.

Scheinert et al. ${ }^{10)}$ reported that the use of distal EPD is safe and effective in patients undergoing high-risk surgeries. Sridhara et al. ${ }^{11)}$ stated that with proper technique, CAS can not only reduce stroke but also prevent stroke-associated cognitive decline, vascular injury, etc. On the contrary, based on the results of the Imaging in Carotid Angioplasty and Risk of Stroke study in patients with echo lucent plaques, de Waard et al. ${ }^{4}$ reported that the use of EPD increases the risk of stroke compared to unprotected CAS $(12.5 \% \text { vs. } 5.2 \% ; \mathrm{p}=0.15)^{1,4)}$. However, in this study, the use of EPD distal filter did not significantly affect the long-term outcomes of CAS.

On the use of EPD to prevent CAS-associated embolism, there are opinions that the use of a familiar, widely used stent rather than selecting the device by matching the lesion and patient characteristics may improve outcomes ${ }^{4)}$. In addition, according to a recent study, the use of closed-cell stents reduce postoperative stroke and the use of small free-cell area stents can reduce the unfavorable post-procedural events ${ }^{12)}$.

This study has several limitations. Because of the retrospective study design, the patient group was not completely designed or conditioned. Moreover, the indication of stent and EPD extension resulted in the use of EPDs in many patients, making it impossible to statistically compare them with the control group that did not use EPD. However, further research is expected in this field because of the recent increase in stent insertion and use of EPD filter to prevent embolism after CAS.

\section{CONCLUSION}

Stent insertion to prevent ipsilateral cerebral infarction presents a higher risk in patients $\geq 80$ years of age and in those with a history of cerebrovascular accidents. Thus, the operating surgeon must be fully aware of these risks. Further studies will be needed to assess the change in complications caused by the use of distal filter device to prevent embolism after CAS and the impact of new technology in treatment of patients with stroke.

\section{CONFLICTS OF INTEREST}

No potential conflict of interest relevant to this article was reported.

\section{REFERENCES}

1. Biasi GM, Froio A, Diethrich EB, Deleo G, Galimberti S, Mingazzini $\mathrm{P}$, et al.: Carotid plaque echolucency increases the risk of stroke in carotid stenting: the Imaging in Carotid Angioplasty and Risk of Stroke (ICAROS) study. Circulation 110:756-762, 2004

2. Bonati LH, Dobson J, Featherstone RL, Ederle J, van der Worp $\mathrm{HB}$, de Borst GJ, et al.: Long-term outcomes after stenting versus endarterectomy for treatment of symptomatic carotid stenosis: the International Carotid Stenting Study (ICSS) randomised trial. Lancet 385:529-538, 2015

3. Brott TG, Hobson RW, 2nd, Howard G, Roubin GS, Clark WM, Brooks W, et al.: Stenting versus endarterectomy for treatment of carotid-artery stenosis. N Engl J Med 363:11-23, 2010

4. de Waard DD, Halliday A, de Borst GJ, Bulbulia R, Huibers A, Casana $\mathrm{R}$, et al.: Choices of stent and cerebral protection in the ongoing ACST-2 trial: A descriptive study. Eur J Vasc Endovasc Surg 53:617-625, 2017

5. Egashira Y, Yoshimura S, Sakai N, Enomoto Y: Real-world experience of carotid artery stentingin Japan: Analysis of 7,134 cases from JR-NET1 and 2 nationwide retrospective multi-center registries. Neurol Med Chir (Tokyo) 54 Suppl 2:32-39, 2014

6. Howard G, Roubin GS, Jansen O, Hendrikse J, Halliday A, Fraedrich G, et al.: Association between age and risk of stroke or death from carotid endarterectomy and carotid stenting: a metaanalysis of pooled patient data from four randomised trials. Lancet 387:1305-1311, 2016

7. Massop D, Dave R, Metzger C, Bachinsky W, Solis M, Shah $\mathrm{R}$, et al:: Stenting and angioplasty with protection in patients at high-risk for endarterectomy: SAPPHIRE worldwide registry first 2,001 patients. Catheter Cardiovasc Interv 73:129-136, 2009

8. Peluso A, Turchino D, Petrone A, Giribono AM, Bracale R, Del Guercio L, et al.: Standard carotid endarterectomy versus carotid artery stenting with closed-cell stent design and distal embolic protection: does the age matter? Transl Med UniSa 19: 60-65, 2019

9. Rothwell PM, Eliasziw M, Gutnikov SA, Fox AJ, Taylor DW, Mayberg MR, et al.: Analysis of pooled data from the randomised controlled trials of endarterectomy for symptomatic carotid stenosis. Lancet 361:107-116, 2003

10. Scheinert D, Reimers B, Cremonesi A, Schmidt A, Sievert H, Rohde $\mathrm{S}$, et al.: Independent modular filter for embolic protection in carotid stenting. Circ Cardiovasc Interv [epub ahead of print, 2017. doi: 10.1161/circinterventions.116.004244] 
11. Sridhara S, Yazdchi S, Alkhatib JT, Berry A, Bokhari IAH: Balloon-assisted tracking of guide extension catheter: A novel technique to retrieve a carotid embolic protection device. Cureus 11:e4045, 2019

12. Stabile E, Giugliano G, Cremonesi A, Bosiers M, Reimers B, Setacci C, et al.: Impact on outcome of different types of carotid stent: results from the European registry of carotid artery stenting. EuroIntervention 12:e265-e270, 2016
13. World Health Organization: Cardiovascular disease. The atlas of heart disease and stroke. Geneva, $\mathrm{CH}$ : World Health Organization, 2004 (https:/www.who.int/cardiovascular_diseases/ resou rces/atlas/en/) [Accessed May 1, 2019]

14. Zahn R, Ischinger T, Mark B, Gass S, Zeymer U, Schmalz W, et al.: Embolic protection devices for carotid artery stenting: is there a difference between filter and distal occlusive devices? J Am Coll Cardiol 45:1769-1774, 2005 